\title{
The Optimal X-Ray Energy Problem in Multi-Phase Flow Metering
}

\author{
Victor R. Bom, Martijn C. Clarijs, Carel W. E. van Eijk, Zvonimir I. Kolar, and Lex M. Scheers
}

\begin{abstract}
When using X-rays in multiphase flow meters operating according to the DEGRA principle, the number of energy values as well as the $\mathrm{X}$-ray energies can in principle be chosen freely. This report assesses the problem of optimization of both the number of energies and the energies themselves for application in the oil industry, where oil, water, and gas mixtures flow through pipes.
\end{abstract}

Index Terms-Fluid flow measurements, nondestructive testing, X-ray applications.

\section{INTRODUCTION}

$\mathbf{P}$ ROBING the contents of pipes by using $\gamma$ or X-ray absorption is a well known technique in the oil industry [1], [2], [3], known as dual energy gamma ray absorption (DEGRA). The radiation can be obtained from a radio nuclide source, or by using an X-ray generator. This latter technique is known as dual energy X-ray absorption (DEXA) [1]. Where the radiation energies from the radio nuclide source are restricted by nature, with an X-ray generator we can choose optimum energies and achieve a better accuracy. This latter advantage establishes a marked difference between DEXA and DEGRA.

In addition an X-ray generator, as it can be switched off, poses less health risk than a source. For devices with a maximum energy below $100 \mathrm{keV}$, generally no heavy licensing is required. The use and transport of radio nuclide sources on the other hand is often subject to very strict licensing. This makes the use of devices based on radio nuclide sources cumbersome and sometimes even impossible.

The problem is to determine the optimum set of energies. As the optimum set will depend on the specific application, we will focus on a flow meter setup as used commonly in the oil industry. There, one seeks to determine the quantities of oil, saline water, and gas that flow though a pipe, given the salinity of the water.

\section{THEORY}

Equations describing the absorption of X-rays by a multicomponent mixture consisting of oil, saline water and gas are derived.

Manuscript received January 29, 2002; revised December 18, 2002

V. R. Bom, M. C. Clarijs, and C. W. E. van Eijk are with the Interfaculty Reactor Institute, Radiation Technology Group, Delft University of Technology, 2629JB Delft, The Netherlands (e-mail: vb@iri.tudelft.nl).

Z. I. Kolar is with the Interfaculty Reactor Institute, Department of Radiochemistry, Delft University of Technology, 2629JB Delft, The Netherlands (e-mail: kolar@iri.tudelft.nl).

L. M. Scheers is with Shell International Exploration and Production, 2280

AB Rijswijk, The Netherlands (e-mail: a.m.scheers@siep.shell.com).

Digital Object Identifier 10.1109/TNS.2003.811279

\section{A. X-Ray Absorption}

The absorption of X-ray radiation in a narrow beam geometry is described by

$$
I=I_{0} e^{-\mu x}
$$

$I$ and $I_{0}$ are the radiation intensities after and before absorption, respectively, $\mu$ is the linear attenuation coefficient, and $x$ is the path length of the radiation (thickness of the absorber). $\mu$ depends on the composition of the absorbing material and on the X-ray energy $E$.

In the case of attenuation by a pipe carrying a multiphase flow formula (1) expands into

$$
I=\Delta I_{0} e^{\Sigma}-\mu_{i}(E) x_{i}, \quad i=1, \ldots, n
$$

where index $i$ indicates the mixture components and $\Delta$ takes account of the small absorption in the windows through which the radiation enters the pipe.

\section{B. Analysis}

By measuring $I$ at $m$ different energies one obtains as many equations

$$
-\ln \left(\frac{I_{j}}{\Delta_{j} I_{0, j}}\right)=\sum_{i=1}^{n} \mu_{i}\left(E_{j}\right) x_{i} \quad \text { for: } j=1,2, \ldots, m .
$$

Since the pipe is filled completely, for a pipe diameter $D$

$$
\sum_{i=1}^{n} x_{i}=D
$$

As the values for $\mu_{i}$ are "known" the set of equations can in principle be solved for the $x_{i} \mathrm{~s}$. When the components are only oil, (saline) water, and gas, and the salinity itself is not considered to be a variable to be solved, the system of equations (3) is a linear system.

By expanding the sum over the components of the mixture and by eliminating $x_{g}$ by using (4), (3) becomes

$$
\begin{aligned}
-\ln \left(\frac{I_{j}}{\Delta_{j} I_{0, j}}\right)-\mu_{g}\left(E_{j}\right) D & =\left(\mu_{o}\left(E_{j}\right)-\mu_{g}\left(E_{j}\right)\right) x_{o} \\
& +\left(\mu_{s w}\left(E_{j}\right)-\mu_{g}\left(E_{j}\right)\right) x_{s w}
\end{aligned}
$$

where the indexes $o, g$ and $s w$ have been used, respectively, for: oil, gas, and saline water. This set of $m$ equations (5) is solved for the two unknowns: $x_{o}$ and $x_{s w}$ with the method of singular value decomposition; $x_{g}$ then follows from (4). Once the path lengths are obtained they are combined with the respective densities to get the amounts of oil/water/gas. 
TABLE I

OPTIMUM ENERGY SETS FOR VARIOUS OIL, WATER, GAS, AND SALINITY Combinations, for Two Energies Used. THE OIL AND Water Path Lengths ARe EXrRessed as a Percentage of $D$. The Low-Energy $E_{l}$, THE High-ENERGY $E_{h}$ ARE EXPRESSED IN KILO-ELECTRON VOLT. THE CORRESPONDING X-RAY SOURCE SPECTRUM INTENSITIES $I_{0, l}$ AND $I_{0, h}$, AND THE TOTAL INTENSITY $I_{0}$, Tot $_{\text {ot }}$ ARE LISTED IN UNITS OF $10^{5}$ PHOTONS/S

\begin{tabular}{c||c||c|cc|cc||c}
\multicolumn{7}{c}{ salinity $50 \mathrm{~kg} N a C l$ per $\mathrm{m}^{3}$} \\
\hline Oil & Water & Error & $E_{l}$ & $I_{0, l}$ & $E_{h}$ & $I_{0, h}$ & $I_{0, \text { Tot }}$ \\
\hline \hline 10 & 10 & 0.014 & 13 & 1.4 & 37 & 6.8 & 10. \\
30 & 10 & 0.015 & 13 & 1.4 & 37 & 6.8 & 10. \\
70 & 10 & 0.018 & 15 & 1.1 & 39 & 7.7 & 11. \\
10 & 30 & 0.017 & 17 & 1.0 & 43 & 9.5 & 13. \\
30 & 30 & 0.019 & 17 & 1.0 & 43 & 9.5 & 13. \\
70 & 30 & 0.023 & 17 & 1.0 & 43 & 9.5 & 13. \\
10 & 70 & 0.028 & 21 & 1.2 & 47 & 11.0 & 16. \\
30 & 70 & 0.031 & 21 & 1.2 & 47 & 11.0 & 16. \\
\hline
\end{tabular}

\begin{tabular}{|c||c||c|cc|cc||c}
\multicolumn{8}{c}{ Salinity $100 \mathrm{~kg} N a C l$ per $\mathrm{m}^{3}$} \\
\hline Oil & Water & Error & $E_{l}$ & $I_{0, l}$ & $E_{h}$ & $I_{0, h}$ & $I_{0, T o t}$ \\
\hline \hline 10 & 10 & 0.013 & 15 & 1.1 & 39 & 7.7 & 11. \\
30 & 10 & 0.014 & 15 & 1.1 & 39 & 7.7 & 11. \\
70 & 10 & 0.017 & 15 & 1.1 & 39 & 7.7 & 11. \\
10 & 30 & 0.017 & 17 & 1.0 & 43 & 9.5 & 13. \\
30 & 30 & 0.018 & 17 & 1.0 & 43 & 9.5 & 13. \\
70 & 30 & 0.022 & 19 & 1.1 & 45 & 10.0 & 14. \\
10 & 70 & 0.028 & 21 & 1.2 & 49 & 12.0 & 17. \\
30 & 70 & 0.031 & 21 & 1.2 & 49 & 12.0 & 17. \\
\hline
\end{tabular}

\section{OPTIMUM ENERGIES}

When the intensities and attenuation coefficients are known with infinite accuracy, (5) will give the correct oil and water path lengths. However, in reality the intensities are not known with mathematical accuracy. First, the intensities will show a statistical uncertainty due to the nature of radiation processes and, second, the intensities can have a systematic uncertainty for instance related to the way in which the data are extracted from the measured X-ray spectrum.

When in the analysis intensities are used that slightly deviate from the infinite accurate values path lengths will result that deviate from the correct values. We define the oil path length error as the deviation of the derived oil path length from the "true" value. This error is calculated as the square root of the sum of squares of the errors which result from the disturbance of the intensity of every single energy in turn.

The optimum set of energies is defined as those energies for which the above defined oil path length error is minimal. At the optimum, (5) is least sensitive to line intensity deviations. It is irrelevant in this respect whether the deviations are systematic or statistical of nature. The optimum set is determined by calculating the error for all relevant energy sets and then selecting the set with the minimal error.

\section{A. Calculations}

The calculations were done using the cross section tables as well as the material and mixture definition features from the well-established GEANT package [7].

For X-ray absorption calculations only the Compton effect and the photo-electric effect are important. The GEANT cross sections for these effects have an inaccuracy of a few percent for energies between $10 \mathrm{keV}$ and $100 \mathrm{GeV}$.

1) Calculation Specifics: The calculations to determine the oil path length error are done under the following assumptions.

- Pipe diameter: An inner pipe diameter or more precisely a path length of the radiation through the mixture of $4 \mathrm{~cm}$ is assumed.

- Scattering: Perfect collimation of the X-ray beam in the setup is assumed, so no scattering is taken into account.

- Detector: A silicon detector with a thickness of $500 \mu \mathrm{m}$ is assumed because it is the most suitable detector for the application. A thinner less efficient detector would require higher intensities, especially for the high-energy X-ray. The effect on the X-ray energies would be small. An alternative would be a $\mathrm{CdZn}(\mathrm{Te})$ detector. These detectors exhibit long tails at the low-energy side of the peak response. This leads to a higher background and to an increased uncertainty in the peak intensity:

- peak width is supposed to be $2 \mathrm{keV}$ at full width at half maximum (FWHM) and $4 \mathrm{keV}$ at the base of the peak, independent of energy;

- the detector efficiency is based on pure $\mathrm{Si}$;

-the resolution line width is ignored, as far as spectrum shape is concerned;

-because of the small detector size, multiple Compton scattering in the detector is ignored;

- Si escape peaks in the detector are not taken into account.

- Walls: To allow the X-rays to enter the oil/water/gas mixture windows of a hydrocarbon material with a density of $1.2 \mathrm{~g} / \mathrm{cm}^{3}$ are assumed in the steel pipe with a total thickness of $8 \mathrm{~mm}$.

- Compton edges: Problems in the determination of the attenuation which may arise when the photo peak from one fluorescence line ends up on top of the Compton edge of another fluorescence line, are ignored.

- Salinity: The salinity of the water component is assumed to be caused only by $\mathrm{NaCl}$.

\section{B. Source Spectrum}

A number of spectra play a role in the calculations:

Source spectrum: The X-ray spectrum that emanates from the slanted foils by the fluorescence mechanisms.

Pipe spectrum: The spectrum after absorption by the pipe windows and the pipe contents.

Detector spectrum: The calculated spectrum as it would be detected by the Si detector. This includes the detector efficiency.

For the sake of the calculations the source spectrum is constructed as follows:

1) detector spectrum is constructed, consisting only of a number of mono energetic lines with equal intensity;

2) source spectrum is calculated from this detector spectrum, assuming an empty pipe, and using the detector efficiency due to the photo effect alone;

3) results from [5] show that a constant background up to $100 \mathrm{keV}$ is present in the X-ray spectra emerging from the slanted foils; therefore a continuous spectrum independent of energy is added to the source spectrum, with a total intensity of $20 \%$ of the total source spectrum; 
TABLE II

Optimum Energy Sets for Various Oil, Water, Gas, And Salinity Combinations, For Three Energies Used. The Low-EnERgy $E_{l}$, THE Middle ENERGy $E_{m}$ ARE EXPRESSED In KILO-ELECTRON VOLTS. THE High-ENERGy IS FiXed AT $70 \mathrm{keV}$. THE CORRESPONDING X-RAY SOURCE SPECTRUM INTENSITIES $I_{0, l}, I_{0, m}$, AND $I_{0, h}$ AND THE TOTAL INTENSITY $I_{0, \text { Tot }}$ ARE LISTED IN UNITS OF $10^{5}$ PHOTONS/S

\begin{tabular}{c||c||c|cc|cc||c||c|||c|cc|cc||c||c}
\hline \multicolumn{1}{c||}{} & \multicolumn{5}{c||}{ salinity $50 \mathrm{~kg} N a C l$ per $\mathrm{m}^{3}$} & \multicolumn{5}{c||}{ salinity $100 \mathrm{~kg} \mathrm{NaCl} \mathrm{per} \mathrm{m}^{3}$} \\
\hline Oil & Water & Error & $E_{l}$ & $I_{0, l}$ & $E_{m}$ & $I_{0, m}$ & $I_{0, h}$ & $I_{0, T o t}$ & Error & $E_{l}$ & $I_{0, l}$ & $E_{m}$ & $I_{0, m}$ & $I_{0, h}$ & $I_{0, \text { Tot }}$ \\
\hline \hline 10 & 10 & 0.017 & 17 & 0.40 & 43 & 3.4 & 17 & 25 & 0.016 & 17 & 0.40 & 43 & 3.4 & 17 & 25 \\
30 & 10 & 0.019 & 17 & 0.40 & 43 & 3.4 & 17 & 25 & 0.017 & 17 & 0.40 & 45 & 3.8 & 16 & 26 \\
70 & 10 & 0.023 & 17 & 0.40 & 45 & 3.8 & 16 & 26 & 0.021 & 17 & 0.40 & 45 & 3.8 & 16 & 26 \\
10 & 30 & 0.022 & 17 & 0.40 & 45 & 3.8 & 16 & 26 & 0.021 & 19 & 0.43 & 49 & 4.8 & 16 & 26 \\
30 & 30 & 0.025 & 19 & 0.44 & 45 & 3.8 & 16 & 26 & 0.023 & 19 & 0.43 & 49 & 4.8 & 16 & 26 \\
70 & 30 & 0.030 & 19 & 0.43 & 47 & 4.3 & 16 & 26 & 0.028 & 19 & 0.43 & 49 & 4.8 & 16 & 26 \\
10 & 70 & 0.038 & 19 & 0.48 & 23 & 0.7 & 18 & 24 & 0.036 & 21 & 0.56 & 25 & 0.8 & 18 & 24 \\
30 & 70 & 0.041 & 19 & 0.48 & 23 & 0.7 & 18 & 24 & 0.039 & 21 & 0.56 & 25 & 0.8 & 18 & 24 \\
\hline
\end{tabular}

4) pipe spectrum is calculated (empty pipe);

5) detector spectrum is recalculated using a basic detector response function consisting of

a) photo peak;

b) Compton "tail" with a rectangular energy distribution.

6) source spectrum is multiplied with a multiplication factor such that the total content of the detector spectrum becomes $10^{5}$ : the assumed maximum count rate.

Since line intensities are to be determined an energy determination pulse mode counting system is required. This poses limits on the count rate. The empty pipe is used in the construction as this gives the highest count rate, which should still be handled by the detector without too much dead-time loss.

\section{Line Intensity}

Now with the pipe filled, for every oil, water, gas, and salinity combination considered, the detector spectrum is calculated from the source spectrum determined as described above. The same basic detector response function was used. Line intensities are extracted from this detector spectrum.

The line intensity is taken as the peak channel content minus the total background under the peak. The background-per-channel is estimated as half the sum of the contents of the channels adjacent to the peak channel. The total background then amounts to the width of the peak at the base times the background-per-channel. The statistical error in the line intensity is taken equal to the square root of the sum of the line intensity and the total background. No matter how accurate the background can be determined, the statistical uncertainty in one single measurement simply follows the Poisson law.

\section{RESULTS}

Results are shown in Tables I and II. These tables list the optimum sets of energies and the corresponding source intensities, for values for oil-, water-, and gas-path lengths, expressed as percentages of the pipe diameter $D$, which cover the normal range of a flow meter for oil industry application, and for two representative salinity values. In these tables, "Error" means the absolute deviation as defined in Section III. All intensities are quoted divided by $10^{5}$ photons/s; the energies are expressed in kilo-electron volts.
We did calculations for the case of two energies and of three energies. In the three-energy case the uppermost energy was fixed at $70 \mathrm{keV}$. At this energy the energy dependence of the Compton cross sections for all substances used become nearly identical. This means that X-rays with this high an energy are not very well suited to distinguish between the components of the pipe mixture. Therefore, the energy of this high-energy $\mathrm{X}$-ray will have little influence on the optimum values of the other two X-rays.

So, for an oil/water combination of 10/70 the oil path length is $0.10(10 \%)$, and the $1 \sigma$ statistics error in the results of the analysis is 0.028 . The analyzed oil path length or fraction thus will vary between $7.2 \%(10-2.8)$ and $12.8 \%(10+2.8)$. As can be seen from the tables, the error at the optimum energy combinations increases with increasing absorption of the mixture; however, the salinity is not playing an important role in this.

With increasing absorption (more water) the lowest energy of the optimum increases. For the two-energies case the highest energy of the pair also increases with increasing absorption. But for the three-energies scenario we see that the middle energy tends to approach the lowest energy with increasing absorption; effectively reducing to the two-energies case!

Furthermore, we see that the error in the two-energies case is significantly lower than in the case of three energies. Also the energies are slightly lower.

\section{A. X-Ray Spectrum From the Detector}

An example of a calculated $\mathrm{X}$-ray spectrum from the detector, is shown in Fig. 1. The Compton edge of the $42-\mathrm{keV}$ line can be clearly seen around $5 \mathrm{keV}$; the Compton edge of the $21-\mathrm{keV}$ line is at the intensity axis. The constant background part of the $\mathrm{X}$-ray spectrum gives rise to Compton interactions as well, resulting in a continuous Compton spectrum, which shows clearly below $15 \mathrm{keV}$.

The Compton continua of the fluorescence lines will not interfere in this case with the peak analysis because they are in general below about $15 \mathrm{keV}$.

The background under the peaks is due to the background in the source spectrum. It shows an exponential decrease with increasing energy because the detector efficiency decreases with increasing energy. At the low-energy side the background decreases because of absorption in the low $\mathrm{Z}$ window in the pipe walls and in the pipe contents. 


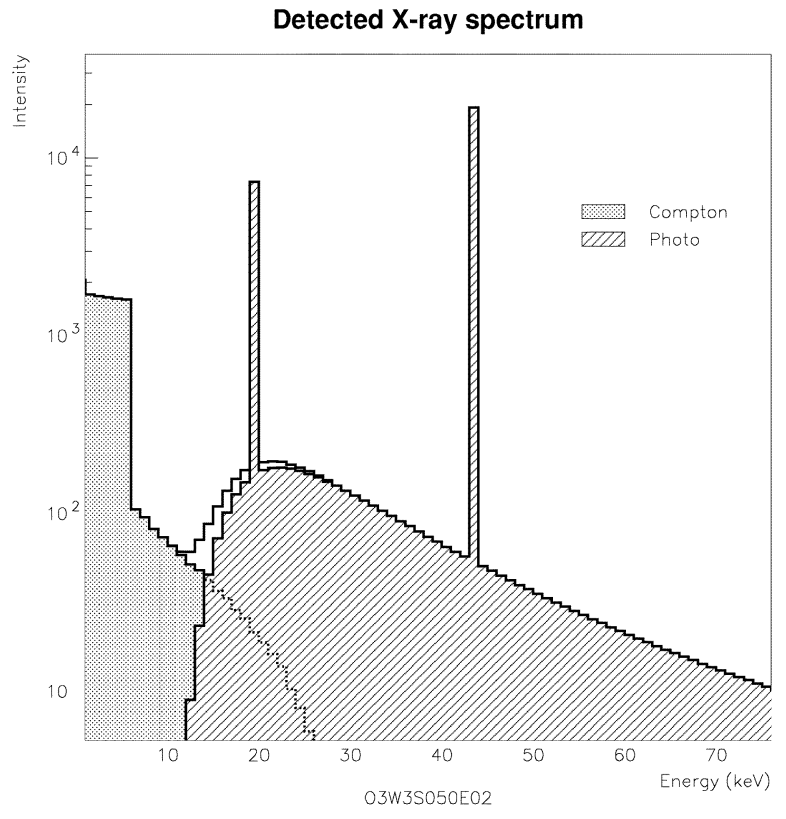

Fig. 1. The result of the calculation of the X-ray spectrum from the silicon detector, for a mixture of $30 \mathrm{vol} \%$ oil, $30 \mathrm{vol} \%$ saline water with a salinity of $50 \mathrm{~kg} / \mathrm{m}^{3}(\mathrm{NaCl})$, and $40 \mathrm{vol} \%$ gas.

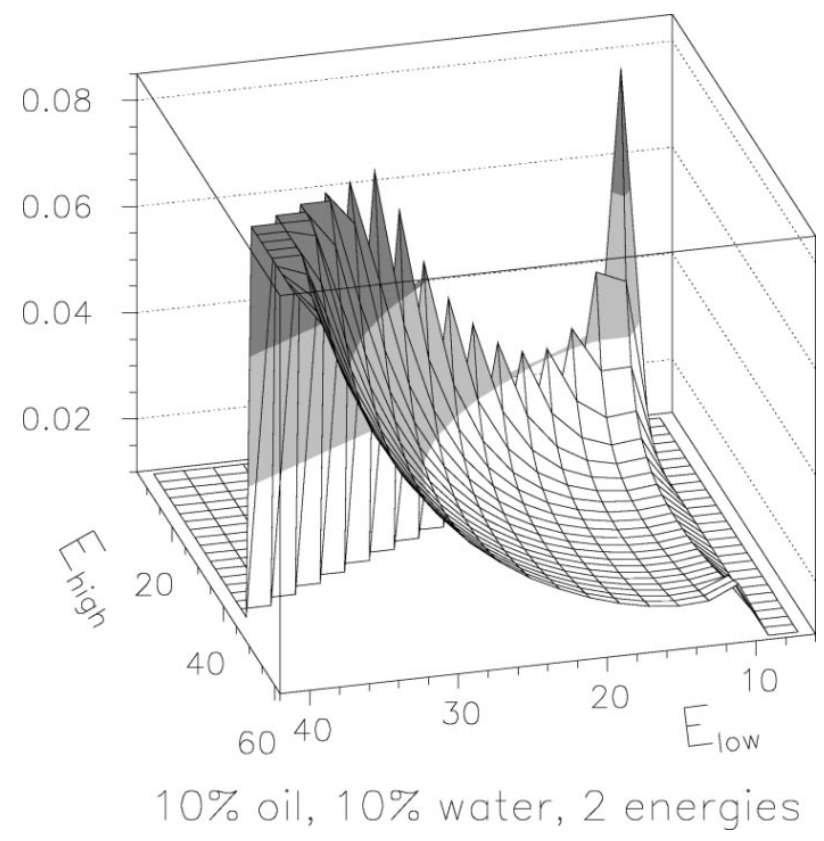

Fig. 2. Oil path length error as function of the energies for the dual energy case. A low absorption mixture is used; the salinity is $50 \mathrm{~kg} / \mathrm{m}^{3}$.

\section{B. The Oil Path Length Error}

Figs. 2 and 3 show the oil path length error when using two energies as a function of the two energies, for two selected mixture compositions, one with a low and one with a high absorption. The salinity in both cases is $50 \mathrm{~kg} / \mathrm{m}^{3}$. The diagonal where $E_{\text {low }}=E_{\text {high }}$ is an axis of symmetry since the high and low energy might be exchanged. The data on one side of the diagonal are shown as zero for clarity.

At low absorption (Fig. 2) a minimum error is located around the point $\{14,40\} \mathrm{keV}$. At high absorption we see that the error

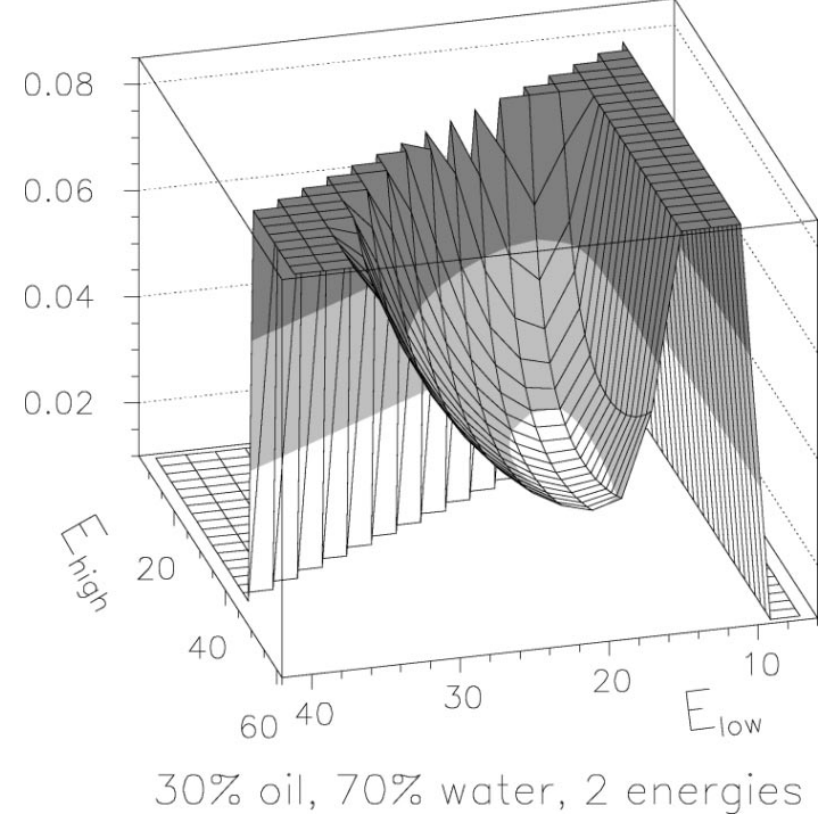

Fig. 3. Oil path length error as function of the energies for the dual energy case. A high absorption mixture is used; the salinity is $50 \mathrm{~kg} / \mathrm{m}^{3}$.

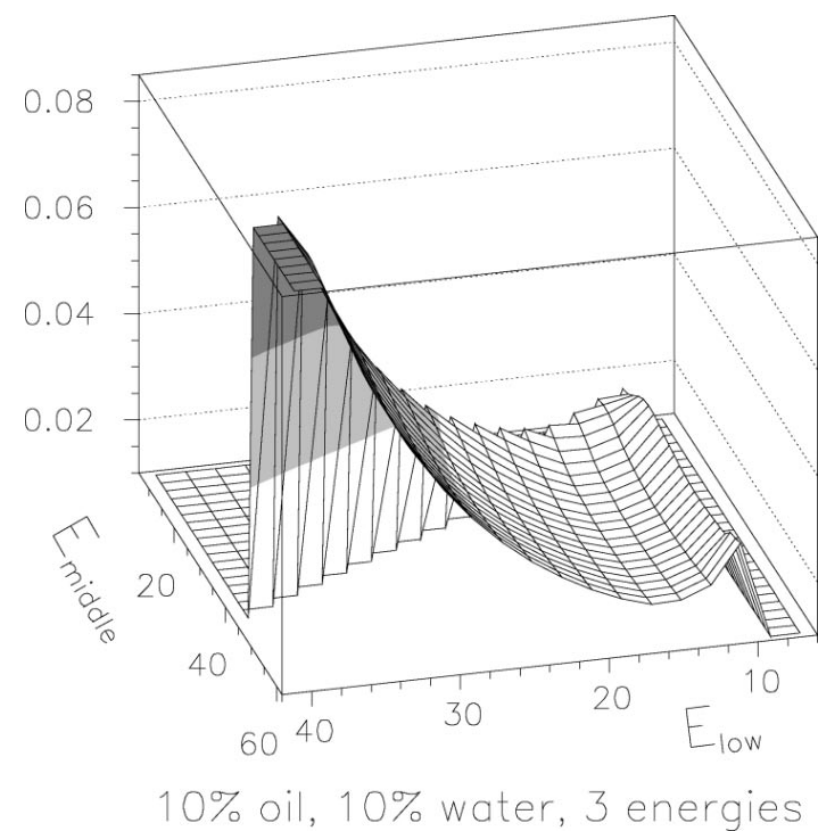

Fig. 4. Oil path length error as function of the two lowest energies for the triple energy scenario. The third energy is set to $70 \mathrm{keV}$. A low absorption mixture is used; the salinity is $50 \mathrm{~kg} / \mathrm{m}^{3}$.

minimum has shifted toward higher energies: $\{20,45\}$, keV, and also that the oil path length error at the minimum has increased. This is of course the same result as was already seen from the Tables I and II.

Figs. 4 and 5 present the same information, but now for the triple-energy scenario. A tendency comparable to the dual-energy case is observed: an upward shift of the energies as the absorption of the mixture increases, accompanied by an increase of the oil path length error at the minimum. In addition it is clear that the value of the oil path length error depends only weakly on one of the energies. For the high absorption case Fig. 5 the oil 


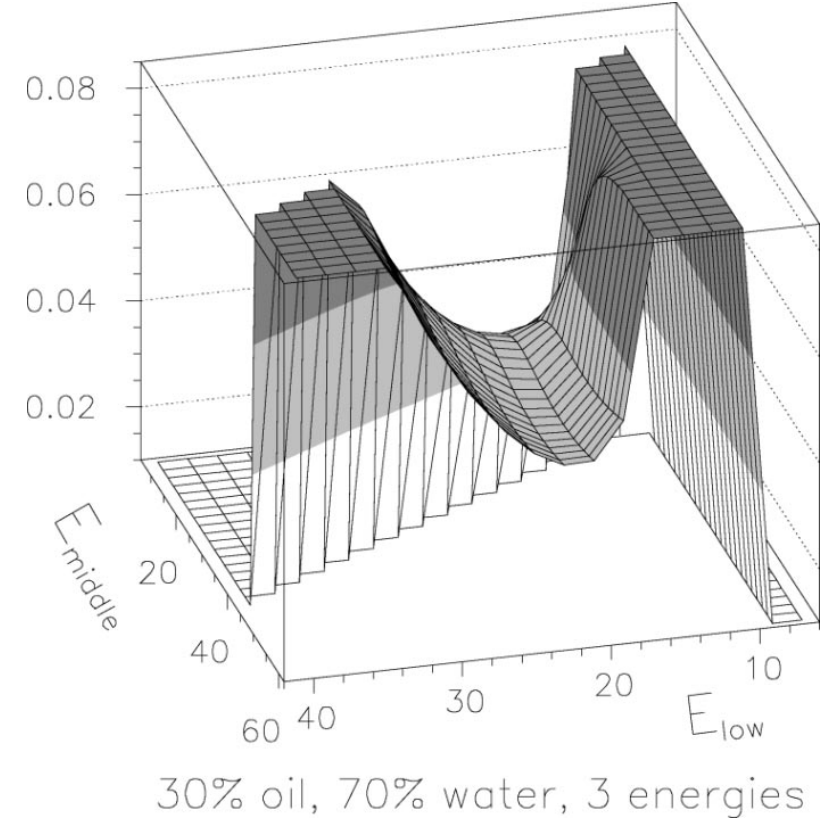

Fig. 5. Oil path length error as function of the two lowest energies for the triple-energy scenario. The third energy is set to $70 \mathrm{keV}$. A high-absorption mixture is used; the salinity is $50 \mathrm{~kg} / \mathrm{m}^{3}$.

path length error minimum even shows the tendency to move toward the diagonal, where the low and middle energies are equal. This means in fact a reduction to the dual-energy scenario!

Focusing on the dual-energy case Fig. 3, we see that for increasing $E_{\text {high }}$, while keeping $E_{\text {low }}$ constant, the oil path length error first decreases and than increases again. This effect can be explained bythe following:

- as $E_{\text {high }}$ is low, the energies are too close together, resulting in a large error;

- as $E_{\text {high }}$ is high, the absorption is less specific as the Compton effect starts dominating.

This same pattern shows up at all $E_{\text {low }}$, but the oil path length error values change: the oil path length error is high for low and high $E_{\text {low }}$, and low for medium $E_{\text {low }}$. The explanation is that

- as $E_{\text {low }}$ is low, the absorption in the mixture is too great, resulting in bad statistics;

- as $E_{\text {low }}$ is high again, absorption gets less specific.

Going from the low absorption mixture to the high absorption mixture the oil error increases because higher absorption leads to smaller line intensities and thus to worse statistics. These arguments explain the existence of a minimum error.

\section{Other Sorts of Deviations}

Up to now, the statistical error has been used for the deviation: the amount with which to disturb the line intensity. This is a choice with statistics in mind, the argument being that the position of the minimum does not depend on the way the line intensities are disturbed.
We also determined oil path length errors using other sorts of deviations

- disturbance of the line intensities by $1 \%$ of the peak content - this kind of disturbance indeed shows a behavior of the minimum which is quite comparable to the behavior as discussed in Section IV-B;

- disturbance of the line intensities by 100 counts - this shows a minimum which moves rapidly to high energies; also the value of the oil path length error at the minimum rises very fast, therefore, this latter approach does not seem a realistic approach to the problem of determination of the optimum set of energies.

\section{CONCLUSION}

It is easy to choose the set of energies if the composition of the mixture in the pipe is known. However, one may want to cover a certain range of mixtures. It can be shown that for the dualenergy case the combination $\{20,46\} \mathrm{keV}$ or $\{22,50\} \mathrm{keV}$ is best for the total range of mixture compositions.

When three energies are preferred, it appears that the combination $\{18,46,70\} \mathrm{keV}$ may be overall best, but it is always worse than the dual-energy scenario. By choosing $\{16,42,70\}$, at least the low absorption mixtures can be measured with about comparable accuracy as in the dual $\{20,46\} \mathrm{keV}$ case. As the absorption increases though, the error for the triple energy scenario rapidly becomes much greater than for the dual-energy case and, it does not help to drop the lowest line of the trio in the analysis. The problem is that the highest energy we are using ( $70 \mathrm{keV}$ ) is very far from the optimum $E_{\text {high }}$ of the dual-energy case.

\section{REFERENCES}

[1] A. M. Scheers and W. F. J. Slijkerman, "Multiphase flow measurement using multiple energy gamma ray absorption measurement,", Denver, CO, SPE 36593 , Oct. 1995.

[2] H. van Santen, Z. Kolar, and A. M. Scheers, "Photon selection for dual energy gamma and/or X-ray absorption composition measurement in oil/water/gas mixtures," Nucl. Geophys., vol. 9, no. 3, pp. 193-202, 1995.

[3] V. R. Bom, M. C. Clarijs, C. W. E. van Eijk, Z. I. Kolar, J. Frieling, A. M. Scheers, and G. Miller, "Accuracy aspects in multiphase flow metering using X-ray transmission," in Proc. Conf. IEEE 2000, vol. 1(1008), Lyon, France.

[4] M. B. Hoppenbrouwers, "Design of a multi phase composition meter for oil-water-gas mixtures based on dual energy X-ray transmission," Stan Ackermans Inst., Eindhoven, The Netherlands, STUDREP.95.081, Jan. 1996.

[5] M. C. Clarijs, "Design studies on cardiovascular and intraoperative brachytherapy and multiphase flow metering, new applications of radiation in medicine and industry," Ph.D. dissertation, Delft Univ. Press, Delft, The Netherlands.

[6] M. C. Clarijs, V. R. Bom, C. W. E. van Eijk, Z. I. Kolar, J. Frieling, A. M. Scheers, and G. Miller, "X-ray spectrum generation for a multi phase flow meter," IEEE Trans. Nucl. Sci., vol. 50, pp. 713-717, June 2003.

[7] R. Brun and F. Carminati, GEANT Detector Description and Simulation Tool, ser. CERN Program Library Long Writeup W5013, Oct. 1994.

[8] G. F. Knoll, Radiation Detection and Measurement, New York: Wiley, 1989. 